\title{
Bruno Bernard Heim, Coutumes et droits héraldiques de l'Église
}

Préface par Donald Lindsay Galbreath.

Précédé de Édouard BOUYÉ, L'Église en armes

Paris, Beauchesne, coll. «Bibliothèque Beauchesne. Religions Sociétés

Politique », 2014, XXXV-198 p.

Notes bibliographiques, index, illustrations hors-texte, annexe.

\section{Paul Airiau}

\section{(2) OpenEdition Journals}

\section{Édition électronique}

URL : http://journals.openedition.org/assr/26448

DOI : $10.4000 /$ assr.26448

ISSN : $1777-5825$

Éditeur

Éditions de l'EHESS

Édition imprimée

Date de publication : 31 décembre 2014

Pagination : 211

ISBN : 978-2-7132-2467-6

ISSN : 0335-5985

Référence électronique

Paul Airiau, «Bruno Bernard Heim, Coutumes et droits héraldiques de l'Église », Archives de sciences sociales des religions [En ligne], 168 | 2014, mis en ligne le 29 avril 2015, consulté le 22 septembre 2020. URL : http://journals.openedition.org/assr/26448 ; DOI : https://doi.org/10.4000/assr.26448

Ce document a été généré automatiquement le 22 septembre 2020.

(C) Archives de sciences sociales des religions 


\section{Bruno Bernard Heim, Coutumes et droits héraldiques de l'Église}

Préface par Donald Lindsay Galbreath.

Précédé de Édouard BOUYÉ, L'Église en armes

Paris, Beauchesne, coll. «Bibliothèque Beauchesne. Religions Sociétés

Politique ", 2014, XXXV-198 p.

Notes bibliographiques, index, illustrations hors-texte, annexe.

Paul Airiau

\section{RÉFÉRENCE}

Bruno Bernard Heim, Coutumes et droits héraldiques de l'Église, Préface par Donald

Lindsay Galbreath.

Précédé de Édouard BOUYÉ, L'Église en armes

Paris, Beauchesne, coll. « Bibliothèque Beauchesne. Religions Sociétés Politique », 2014, XXXV-198 p.

Notes bibliographiques, index, illustrations hors-texte, annexe. 
1 Mais pourquoi donc les Éditions Beauchesne jugent-elles utile de rééditer, en 2014, la traduction française de 1949 de l'ouvrage paru en Suisse que le jeune prélat suisse Bruno Bernard Heim (1911-2003) avait consacré en 1947 à l'héraldique ecclésiastique catholique (Wappenbrauch und Wappenrecht in der Kirche, Olten, O. Walter)? Et, de plus, qu'est-ce qui peut justifier qu'un recenseur prenne la peine de traiter, en long, en large et en travers, d'une telle lubie - hormis son indécrottable affection pour les sujets et personnages quelque peu marginaux aux yeux des gens si sensés qui peuplent le monde universitaire?

2 Peut-on cependant parler de sujet anecdotique, lorsque l'on découvre que

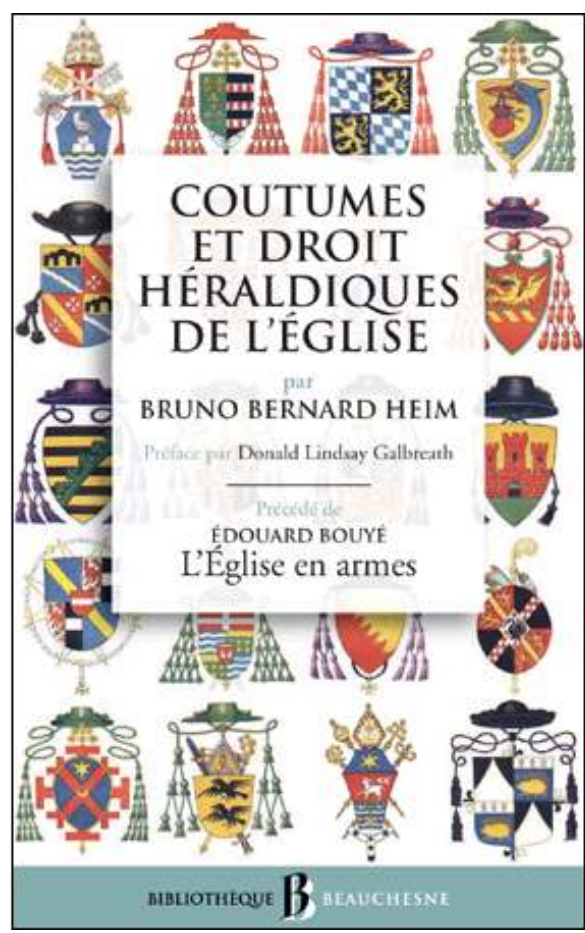
l'ouvrage du camérier secret surnuméraire Heim reçut le prix Lange de l'Académie française en 1950 (certes, l'Académie française, un prix, en 1950...) ; qu'il mena une carrière diplomatique qui ne fut pas sans couleurs ni responsabilités : Académie des Nobles (1942), docteur en droit canonique (1946), attaché à la nonciature de Paris (1947), puis à celles de Vienne (1951) et Bonn (1956), délégué apostolique en Scandinavie (1960), pro-nonce en Finlande (1966), pro-nonce en Égypte (1969), délégué apostolique en Grande-Bretagne (1973) où il devient pro-nonce (1982) avant sa retraite (1985) - je conseille hautement la lecture des nécrologies que le Guardian et le Telegraph lui consacrèrent : Peter Stanford, «The Most Rev Bruno Heim. Indiscreet Catholic diplomat who enjoyed repairing the Reformation rift ", http://www.theguardian.com/news/2003/mar/25/ guardianobituaries.religion et "The Most Reverend Bruno Heim ", http:// www.telegraph.co.uk/news/obituaries/1425427/The-Most-Reverend-Bruno-

Heim.html) ; qu'il dessina les armes des papes de Jean XXIII à Jean-Paul II, sans compter celles d'un grand nombre d'ecclésiastiques; qu'il fut un des grands spécialistes de l'héraldique ecclésiastique et un membre important du milieu héraldique mondial. Certes. Mais a-t-on alors affaire à autre chose qu'à un prélat mondain et diplomate se satisfaisant de byzantines discussions?

Oui, il faut le dire. Ne serait-ce que parce que l'activité de Mgr Heim, en son surgissement originel avec ce livre puis en son déploiement jusqu'à sa mort, montre que l'héraldique ecclésiastique n'est pas, au moins en dehors du monde français, une réalité morte. Elle est au contraire bien vivace dans les mondes anglo-saxons et germaniques - et les capacités héraldiques $\mathrm{du}$ pro-nonce servirent très vraisemblablement à son insertion dans la société britannique (Peter Bander van Duren, "The renaissance of Catholic heraldry 1945-1980 ", Coat of Arms, n 112, hiver 1979, http://www.theheraldrysociety.com/articles/ecclesiastical/ catholic_heraldry.htm). Qu'une telle réalité s'affirme, alors que la somptuosité et la majesté ont régressé dans le catholicisme depuis Vatican II, ne peut qu'interroger 
l'historien soucieux des signes des temps. La profanisation-banalisation de la majesté étatique et la «starification »-«peopolisation» du personnel politique dans les États contemporains depuis une trentaine d'années, qui s'est déployée dans l'Église catholique avec la simplification du décorum curial, la réforme (quasi-suppression) de la Cour pontificale et l'accès au trône papal d'un Karol Wojtyla exerçant sa fonction en rompant assez nettement avec le hiératisme sacral, ne doit point faire illusion. La représentation de soi-même qu'est l'héraldique ecclésiastique n'a pas disparu, et, même, s'est renouvelée depuis Pie XII en partie grâce à l'action de Mgr Heim.

Le rôle du prélat suisse fut ici en effet double. Il proposa d'abord une synthèse accessible sur les principes de l'héraldique ecclésiastique. Son ouvrage dissocie nettement les armes (l'écu) d'un ecclésiastique, pour lesquelles l'Église catholique ne dispose d'aucune règle et n'exerce aucune compétence propre, de leurs timbres (les symboles encadrant d'une manière ou d'une autre les armes : tiare, mitre, crosse, croix, chapeau...), qui relèvent eux en revanche de l'Église catholique, puisqu'ils indiquent une appartenance à la hiérarchie et un degré en celle-ci. Cependant, aucune législation homogène n'a jamais été produite. Aussi, le code de droit canonique de 1917 (et celui de 1983 a fortiori) n'indiquant aucune règle, il s'agit d'un droit coutumier, à combiner avec les prescriptions papales, les décisions curiales et les pratiques de la Cour romaine, le tout étant étalé sur trois siècles et n'ayant pas été aboli par la codification de 1917. Travail de canoniste et d'héraldiste donc, que celui de Mgr Heim, qui aboutit à une affirmation simple: «la législation héraldique de l'Église [...] ne vise qu'à obtenir la simplicité et la claire distinction de grades hiérarchiques, ainsi qu'à empêcher l'emploi illégal et toute usurpation des insignes » (p. 157).

5 Mais travail d'esthète également, et c'est ici que se développa le second rôle du prélat. En effet, ne se contentant pas d'ordonner la législation héraldique ecclésiastique à partir de ses principes, il propose aussi des conseils pour la création d'armoiries ecclésiastiques. Il appelle d'abord les ecclésiastiques sans armes et qui doivent (ou veulent) en créer (par exemple les évêques nouvellement nommés) à faire appel à de véritables spécialistes du blason, à délaisser les armes parlantes inspirées d'images pieuses et à ne pas surcharger l'écu. Il « faut choisir des armes à la fois simples et originales qui remplissent le champ de l'écu d'une façon harmonieuse et proportionnée » (p.154). Cet appel aux spécialistes s'accompagne d'une articulation à l'artistique : "la liberté de l'artiste authentique ne peut - et nulle part autant que dans ce domaine - s'épanouir que sur le terrain d'une connaissance parfaite du sujet " (p. 156). Ainsi aboutit-il à défendre le «beau réalisme vivant de l'origine» de l'héraldique contre « la mignardise et la fantaisie, souvent inspirée par des tendances fastueuses" (p.59). La simplicité est le maître mot, contre «le faste extravagant, l'accumulation inutile et gauche de timbres et autre accessoires» (p. 115). La «noble simplicité » (p.116), la «simplicité majestueuse » (p.115) doivent devenir l'objectif d'un « art héraldique noble et clair, épuré, approprié à son but, s'inspirant de nouveau des formes primitives. » (p. 117), correspondant en fait « au goût moderne» (p. 117). Et Mgr Heim de prêcher d'exemple en proposant 19 planches comprenant des armes timbrées qu'il a lui-même dessinées, et qu'il commente. Cette capacité artistique explique aussi qu'il ait pu réaliser les armoiries papales, et moult autres (3 000 à suivre Peter Bander van Duren, art. cit.).

6 Aussi peut-on désormais historiciser pleinement l'ouvrage du jeune diplomate en poste à Paris. En effet, il propose rien moins qu'un retour par-delà le médiévalisme du XIX 
siècle, par-delà l'héraldique baroque, classique, renaissante, à une esthétique médiévale, ressaisie et retravaillée pour l'actualiser. Comment ne pas rapprocher alors cette adaptation mesurée de celles que Pie XII réalisa, par exemple avec les instituts séculiers, l'application pratique en morale de la théorie des actions à double effet, et l'intégration de la démocratie dans le corpus théologico-politique magistériel? Comment ne pas y voir le prolongement de la remise en cause de l'art sulpicien massivement diffusé au profit de formes esthétiques beaucoup plus proches de ce qui se développe dans l'art sacré dans les années 1920-1930, et qui, en liturgie, conduit par exemple au développement d'une paramentique gothique ? À ce titre, au moins, il faut lire Mgr Heim. Il faut le lire aussi parce qu'il montre, avec ce cas de l'héraldique, que la gestion purement canonique de l'Église, qu'une administration réduite à l'édiction de règles à appliquer strictement, n'a jamais existé, y compris dans la symbolique la plus visible. En insistant sur la dimension coutumière et sur les limites de la régulation curiale, l'ouvrage manifeste (et plaide en faveur de) la souplesse relative du gouvernement. L'ensemble de la vie catholique n'a pas été saisi par le code de droit canonique, et il reste de la place pour des réalités extérieures. Certes, l'Église y aura son mot à dire, pour ce qui la concerne, c'est-à-dire les signes hiérarchiques extérieurs aux écus, mais l'autonomie des réalités terrestres est là, et bien là, sans que cela soit dommage. Difficile de dire qu'on anticipe Vatican II : ce serait quelque peu brusquer les choses et tordre la réalité. Mais il n'en reste pas moins que, dans le monde curial et canoniste, au sortir de la guerre, les choses sont plus complexes qu'on pouvait le vivre ou le penser, ou qu'on a pu le reconstruire de mémoire.

7 Dernière manière d'historiciser Mgr Heim : L'Église en armes, d'Édouard Bouyé, qui précède le travail du jeune prélat. L'actuel directeur des archives départementales de Côte-d'Or (il dirigeait celles du Cantal lorsque son texte a été rédigé) est lui-même spécialiste d'héraldique ecclésiastique médiévale. Son texte ouvre excellemment la réédition. Il introduit à l'homme et à son travail, en soulignant des apports que le contemporanéiste ne pourrait voir. Il utilise les travaux d'histoire culturelle de l'héraldique et des couleurs de Michel Pastoureau pour expliquer la couleur verte (et non violette) du chapeau épiscopal et l'appropriation du violet par le costume lui aussi épiscopal. Il exploite les archives du cardinal Jacques Martin (1908-1992), ancien préfet de la Maison Pontificale, pour souligner l'importance de la passion héraldique chez les clercs. Il utilise sa science héraldique et historique pour attirer l'attention sur l'usage des symboles papaux chez Benoît XVI. Bref, il multiplie les pistes pour illustrer l'intérêt et l'actualité de Mgr Heim.

Et l'on ne peut que le suivre, tant les symboles demeurent puissants. Il n'est pour s'en convaincre que de visiter le blog de l'Argentin Alejandro Pomar, amateur d'héraldique, et ses pages consacrées aux « armoiries pseudo-ecclésiastiques » qu'il croise de-ci de-là (http://heraldicaargentina.blogspot.fr/search/label/

her\%C3\%A1ldica\%20pseudoeclesi\%C3\%A1stica). Les armes continuent encore et toujours à être des instruments de légitimation pour les clercs vagus, les entrepreneurs de religion, les papes alternatifs, et tous les autres. 